\title{
Role of CD8 Cytotoxic T Lymphocytes in Hepatocellular Carcinoma: An Immunohistochemical Study
}

\author{
ASMAA G. ABDOU, M.D.*; HALA S. EL-REBEY, M.D.*; NANIS S. HOLAH, M.D.*; \\ MERVAT S. SULTAN, M.D.** and SHYMAA H. IBRAHIM, M.Sc.** \\ The Department of Pathology, Faculty of Medicine* and National Liver Institute**, Menoufia University, Egypt
}

\begin{abstract}
Background: Hepatocellular Carcinoma (HCC) is the most common primary malignancy of the liver in adults accounting for $85 \%-90 \%$ of liver tumors. It represents the sixth most common malignancy and the fourth leading cause of cancer-related death worldwide. There is an important role of CD8+ tumor infiltrating lymphocytes in host immune defense against tumour progression. Immunotherapy, with modern pharmacologic developments, is a new direction in cancer therapy and therefore immunobiology of hepatocarcinogenesis is under investigation.
\end{abstract}

Aim of Study: Study expression of CD8 in Hepatocellular carcinoma and its correlation with clinicopathological parameters and explore its effect on HCC prognosis.

Material and Methods: This study included 112 hepatocellular carcinoma cases obtained from the archival material of Pathology Department, National Liver Institute, Menoufia University, between 2010 and 2017. All cases were stained for CD8 antibody. Survival data were available for all HCC cases.

Results: CD8 was expressed in $110 \mathrm{HCC}$ cases (98.2\%). There was a statistical significant association between high CD8 percentage and non-cirrhotic adjacent liver $(p=0.035)$. On the other hand, there was a significant association between high CD8 percentage and large tumor size $(<5 \mathrm{~cm})(p=0.015)$. There was no statistically significant association between CD8 expression and overall survival or recurrence.

Conclusion: We concluded that high CD8 might serve as bad prognostic parameter in $\mathrm{HCC}$ as it is associated with noncirrhotic liver and large tumor size but it doesn't affect HCC overall survival or recurrence.

Key Words: CD8 - HCC - Immunohistochemistry - Prognosis.

\section{Introduction}

HEPATOCELLULAR Carcinoma (HCC) is the most common primary malignancy of the liver in adults accounting for $85 \%-90 \%$ of liver tumors [1]

Correspondence to: Dr. Shymaa H. Ibrahim, E-Mail: dr.shymaa.hany@.gmail.com
It represents the sixth most common malignancy and the fourth leading cause of cancer-related death worldwide $[2,3]$.

In Egypt, liver cancer forms $11.75 \%$ of the malignancies of all digestive organs and $1.68 \%$ of the total malignancies. HCC constitutes $78.64 \%$ of all liver tumors among Egyptians [4]. HCC is the most common cancer in males and the second in females $[\mathbf{5 , 6}]$.

Normally, stroma maintains the tissue homeostasis and acts as a barrier toward tumor formation; however, when a cell starts to be cancerous, its surrounding matrix changes in a way to support cancer development $[\mathbf{7 , 8}]$. This modified stroma around the malignant cells is termed Tumor Microenvironment (TME) [9].

Tumor Infiltrating Lymphocytes (TILs) are a class of cells that shape the tumor microenvironment and therefore affect carcinogenesis [10]. TILs are considered manifestations of host immune reactions against cancers. Patients with a prominent lymphocyte infiltrate, especially $\mathrm{T}$ lymphocytes, who underwent resection for HCC, have reduced recurrence and better survival [11]

There is an important role of CD8+ tumor infiltrating lymphocytes in host immune defense against tumour progression. Studies have indicated a positive correlation between an increased number of CD8+ TILs and the occurrence of tumour cell apoptosis with inhibition of tumor progression $[12,13]$

Immunotherapy, with modern pharmacologic developments, is a new direction in cancer therapy and therefore the immunobiology of hepatocarcinogenesis is under investigation $[\mathbf{1 0 , 1 4 ]}$ 
The aim of this study is to evaluate the expression of CD8 in HCC cases and its correlation with clinicopathological parameters and explore its effect on HCC prognosis.

\section{Material and Methods}

This retrospective case study included 112 Hepatocellular Carcinoma (HCC) cases. All specimens were obtained from Egyptian patients either by partial hepatectomy or total hepatectomy procedures and retrieved from the archival material of Pathology Department, National Liver Institute, Menoufia University, during the period between 2010 and 2017. They were selected according to the availability of the blocks for serial cutting and examination. Survival data were available for all of the studied HCC cases.

\section{Clinical data:}

Data were collected from patients' medical records including:

- Age: Studied cases were divided into two groups; $<60$ years and $\geq 60$ years [15]

- Gender.

- Laboratory investigations:

- Serological detection of viral etiology by ELISA or PCR.

- Serum Alpha Fetoprotein (AFP) (available for 91 cases only) was recorded as the highest value within the 6 months prior to surgical resection [16]. Patients were divided into two groups; $<200 \mathrm{ng} / \mathrm{ml}$ and $\geq 200 \mathrm{ng} / \mathrm{ml}$ [17] .

- Child pugh classification (available for 88 cases only) was divided into three classes (A, B and C) according to five clinical measures of liver disease (total bilirubin, serum albumin, prothrombin time or INR, ascitis and hepatic encephalopathy) [18-20]

- Radiological investigations: To detect [21]:

- Tumor site: Unilobar or Bilobar.

- Type of treatment: Interferon, Direct Acting Antiviral (DAA) or not received any treatment [22].

- CLIP prognostic score: Available for 88 cases only. It includes the number of nodes, Alphafetoprotein (AFP) level, portal vein thrombosis and Child-Pugh classification. It was classified from $0-5[23,24]$

Histopathological evaluation: From each representative paraffin block of the studied cases,
4 thick sections were cut, stained by haematoxylin and eosin (H \& E) and re-evaluated to confirm the diagnosis and to assess the following:

- Tumor size: The greatest dimension in centimetres. Cases were divided into two groups; tumor size $<5 \mathrm{~cm}$ and $\geq 5 \mathrm{~cm}[25]$

- Tumor focality: Single or multiple [15].

- Clear cell changes: It was assessed subjectively as present or absent.

- Tumor grade $(G)$ : Classic HCC was graded based on the highest grade of tumor differentiation [26] Grade of classic HCC was then lumped into two groups; low grade (G 1-2) and high grade (G 34) $[27,28]$

- Pathological stage: According to American Joint Committee on Cancer (AJCC) staging system, $8^{\text {th }}$ edition [29]. HCC cases were divided into two groups; early stage (T 1-2) and advanced stage (T 3-4) [30]

- Lymphovascular Invasion (LVI): LVI was defined as presence of tumor cells within or adherent to the wall of an unequivocal endothelial-lined vascular space (vascular or lymphatic) [31]

-Adjacent non-tumor liver tissue: Cirrhotic or non cirrhotic [15]

Tissue Microarray Constructing Technique (TMA):

Three tissue cores with a diameter of 1.5 micron from the selected area in the donor block were punched using a manual tissue arrayer's needle (Breecher Instrument, USA) then arrayed on a recipient block. Tissue Microarray Constructing Technique (TMA) map was created indicating the position and origin of each core in the tissue microarray. A control normal tissue was placed in strategic regions throughout the blocks [32]. Five micron thick sections were cut from recipient block and placed on positive charged slides and used for immunohistochemical staining.

\section{Immunohistochemistry:}

Paraffin-embedded tissue sections $(5 \mathrm{~mm})$ were deparaffinized in xylene and rehydrated. The sections were treated with $200 \mathrm{ml}$ of tris-EDTA high PH retrieval solution (Dako, Ref K8000, Glostrup, Denmark) for 20 minutes. Endogenous peroxidase was blocked with peroxidase-blocking reagent. The primary antibody was CD8 [Clone C8/144B, A monoclonal mouse anti-human antibody (Dako Denmark. A/S)] which was ready to use.

A positive reaction was revealed using substratechromogen solution (DAB). The sections were 
then counterstained with Mayer's hematoxylin. Positive controls for the reaction were performed with paraffin-embedded sections of normal human tonsil and negative controls were used for each run of immunohistochemical staining by omitting the primary antibody.

\section{Interpretation of immunostaining results:}

Assessments of immunohistochemical results were determined using a semiquantitative visual approach. Unintentional bias was prevented by coding patients' slides.

Positive cells were identified by the presence of brownish membranous coloration detected by $\mathrm{DAB}$ reaction. The staining intensity was also reported and scored from 0 to $3(0=$ Negative, $1=$ Mild staining, $2=$ Moderate staining and $3=$ Strong staining) for each cells. The staining intensity score 2 and 3 were classified as positive. $20 \%$ was used as cutoff point for percentage of positive lymphocytes and cases were divided into two groups; low percentage (0-20\%) and high percentage (21$100 \%)[15]$

\section{Overall survival and recurrence data:}

Overall survival time and recurrence were available for all 112 patients.

- Overall survival: OS was calculated from the date of surgery to either the date of death or the last follow-up [33].

- Recurrence: The diagnosis of recurrence was based on typical imaging appearance with or without an elevated serum AFP after the date of surgery $[34,35]$.

\section{Statistical analysis:}

Data were fed to the computer and analyzed using Statistical Package for the Social Sciences (SPSS) software package Version 20.0. (Armonk, NY: IBM Corp). Qualitative data were described using number and percent.

Chi-Square $\left(\chi^{2}\right)$ test and Fisher Exact $(\mathrm{FE})$ test were used for categorical variables, to compare between different groups. Mann-Whitney (U) test and Kruskal-Wallis $(\mathrm{H})$ test were used for abnormally distributed quantitative variables, to compare between two or more studied groups. Kaplan Meier test was constructed to differentiate survival and recurrence between compared groups using the log rank test. $p \leq 0.05$ was considered to indicate statistical significance in all tests [36].

\section{Results}

Table (1): Clinicopathological characteristics of the studied HCC cases.

\begin{tabular}{|c|c|c|c|}
\hline \multirow{2}{*}{ Variables } & & \multicolumn{2}{|c|}{$\mathrm{HCC}(\mathrm{No} .=112)$} \\
\hline & & $\frac{\text { No }}{75}$ & $\frac{\%}{67.0}$ \\
\hline Age: & $\begin{array}{l}<60 \\
\geq 60 \\
\text { Min.-max. } \\
\text { Mean } \pm \text { SD. } \\
\text { Median }\end{array}$ & \multicolumn{2}{|c|}{$\begin{array}{c}35.0-75.0 \\
56.50 \pm 7.09 \\
57.0\end{array}$} \\
\hline Gender: & $\begin{array}{l}\text { Male } \\
\text { Female } \\
\text { M:F }\end{array}$ & $\begin{array}{l}91 \\
21 \\
4.3: 1\end{array}$ & $\begin{array}{l}81.2 \\
18.8\end{array}$ \\
\hline Etiology: & $\begin{array}{l}\text { Viral } \\
\text { Non viral }\end{array}$ & $\begin{array}{l}101 \\
11\end{array}$ & $\begin{array}{l}90.2 \\
9.8\end{array}$ \\
\hline$A F P(n g / m l)(N o=91):$ & $\begin{array}{l}\text { Min.-max. } \\
\text { Mean } \pm \text { SD. } \\
\text { Median }\end{array}$ & \multicolumn{2}{|c|}{$\begin{array}{c}1.0-20010.0 \\
930.5 \pm 3070.5 \\
33.0\end{array}$} \\
\hline $\operatorname{AFP}(n g / m l)(N o=91):$ & $\begin{array}{l}<200(\mathrm{ng} / \mathrm{ml}) \\
\geq 200(\mathrm{ng} / \mathrm{ml})\end{array}$ & $\begin{array}{l}71 \\
20\end{array}$ & $\begin{array}{l}78.0 \\
22.0\end{array}$ \\
\hline $\begin{array}{l}\text { Child pugh classification } \\
(N o=88):\end{array}$ & $\begin{array}{l}\text { A } \\
\text { B } \\
\text { C }\end{array}$ & $\begin{array}{l}58 \\
30 \\
0\end{array}$ & $\begin{array}{l}65.9 \\
34.1 \\
0\end{array}$ \\
\hline Focality: & $\begin{array}{l}\text { Single } \\
\text { Multiple }\end{array}$ & $\begin{array}{l}80 \\
32\end{array}$ & $\begin{array}{l}71.4 \\
28.6\end{array}$ \\
\hline Site: & $\begin{array}{l}\text { Unilobar } \\
\text { Bilobar }\end{array}$ & $\begin{array}{l}106 \\
6\end{array}$ & $\begin{array}{l}94.6 \\
5.4\end{array}$ \\
\hline Treatment: & $\begin{array}{l}\text { Interferon } \\
\text { DAA } \\
\text { No }\end{array}$ & $\begin{array}{l}24 \\
49 \\
39\end{array}$ & $\begin{array}{l}21.4 \\
43.8 \\
34.8\end{array}$ \\
\hline $\begin{array}{l}\text { CLIP prognostic score sys- } \\
\text { tem }(N o=88) \text { : }\end{array}$ & $\begin{array}{l}0 \\
1 \\
2 \\
3 \\
4 \\
5\end{array}$ & $\begin{array}{l}20 \\
42 \\
10 \\
11 \\
4 \\
1\end{array}$ & $\begin{array}{l}22.7 \\
47.7 \\
11.4 \\
12.5 \\
4.5 \\
1.2\end{array}$ \\
\hline Size: & $\begin{array}{l}\text { Min.-max. } \\
\text { Mean } \pm \text { SD. } \\
\text { Median } \\
<5 \mathrm{~cm} \\
\geq 5 \mathrm{~cm}\end{array}$ & \multicolumn{2}{|c|}{$\begin{array}{c}1.0-17.0 \\
5.19 \pm 3.11 \\
4.0\end{array}$} \\
\hline Clear changes: & $\begin{array}{l}\text { Yes } \\
\text { No }\end{array}$ & $\begin{array}{l}9 \\
103\end{array}$ & $\begin{array}{l}8.0 \\
92.0\end{array}$ \\
\hline Grade: & $\begin{array}{l}\text { I } \\
\text { II } \\
\text { III } \\
\text { IV }\end{array}$ & $\begin{array}{l}1 \\
76 \\
31 \\
4\end{array}$ & $\begin{array}{l}0.9 \\
67.9 \\
27.6 \\
3.6\end{array}$ \\
\hline Combined grade: & $\begin{array}{l}\mathrm{I} / \mathrm{II} \\
\mathrm{III} / \mathrm{IV}\end{array}$ & $\begin{array}{l}77 \\
35\end{array}$ & $\begin{array}{l}68.8 \\
31.2\end{array}$ \\
\hline Pathological stage: & $\begin{array}{l}\text { T1 } \\
\text { T2 } \\
\text { T3 } \\
\text { T4 }\end{array}$ & $\begin{array}{l}44 \\
55 \\
12 \\
1\end{array}$ & $\begin{array}{l}39.3 \\
49.1 \\
10.7 \\
0.9\end{array}$ \\
\hline $\begin{array}{l}\text { Combined pathologic } \\
\text { stage: }\end{array}$ & $\begin{array}{l}\text { Early (T1/T2) } \\
\text { Advanced (T3/T4) }\end{array}$ & $\begin{array}{l}99 \\
13\end{array}$ & $\begin{array}{l}88.4 \\
11.6\end{array}$ \\
\hline Lymphovascular invasion: & $\begin{array}{l}\text { Present } \\
\text { Absent }\end{array}$ & $\begin{array}{l}49 \\
63\end{array}$ & $\begin{array}{l}43.8 \\
56.2\end{array}$ \\
\hline Adjacent liver: & $\begin{array}{l}\text { Cirrhotic } \\
\text { Non cirrhotic }\end{array}$ & $\begin{array}{l}92 \\
20\end{array}$ & $\begin{array}{l}82.1 \\
17.9\end{array}$ \\
\hline
\end{tabular}

HCC : Hepatocellular Carcinoma. Max. : Maximum.

No : Number. $\quad \mathrm{M} / \mathrm{F}$ : Male to female ratio.

$\%$ : Percentage. AFP : Alpha fetoprotein.

SD : Standard Deviation. DAA : Direct Acting Antiviral.

Min. : Minimum. CLIP : Cancer of the liver Italian score program score. 
Clinicopathological characteristics of the patients: Clinicopathological characteristics of the selected cases are showed in (Table 1).

\section{CD8 immunohistochemical results:}

CD8 was expressed in tumor infiltrating lymphocytes of 110 HCC cases (98.2\%) Fig. (1A), (Table 2).

Table (2): Immunohistochemical expression of CD8 in the studied HCC cases.

\begin{tabular}{|c|c|c|}
\hline \multirow{2}{*}{ Variables } & \multicolumn{2}{|c|}{$\operatorname{HCC}($ No. $=112)$} \\
\hline & No & $\%$ \\
\hline \multicolumn{3}{|l|}{ CD8 expression: } \\
\hline Negative & 2 & 1.8 \\
\hline Positive & 110 & 98.2 \\
\hline \multicolumn{3}{|l|}{ CD8 intensity: } \\
\hline 0 & 2 & 1.7 \\
\hline 1 & 0 & 0.0 \\
\hline 2 & 21 & 18.8 \\
\hline 3 & 89 & 79.5 \\
\hline \multicolumn{3}{|l|}{ Counting score: } \\
\hline 0 & 23 & 20.5 \\
\hline 1 & 35 & 31.3 \\
\hline 2 & 54 & 48.2 \\
\hline \multicolumn{3}{|l|}{ CD8 percentage: } \\
\hline Low percentage $(0-20)$ & 63 & 56.2 \\
\hline High percentage $(21-100)$ & 49 & 43.8 \\
\hline Min.-max. & \multicolumn{2}{|c|}{$0.0-90.0$} \\
\hline Mean \pm SD & \multicolumn{2}{|c|}{$26.96 \pm 22.33$} \\
\hline Median & \multicolumn{2}{|c|}{20.0} \\
\hline
\end{tabular}

HCC : Hepatocellular Carcinoma.

No : Number.

$\% \quad$ : Percentage.

SD : Standard Deviation.

Min. : Minimum.

Max. : Maximum

A statistically significant association was found between high CD8 percentage and bad prognostic parameters as adjacent non cirrhotic liver $(p=0.035)$ (Table 3), Figs. (1B,C). Also, high CD8 percentage was statistically associated with large sized tumors $(<5 \mathrm{~cm})(p=0.015)$ (Table 3).

Using univariate survival analysis, there was no statistically significant association between CD8 expression and overall survival or recurrence (Table 4).
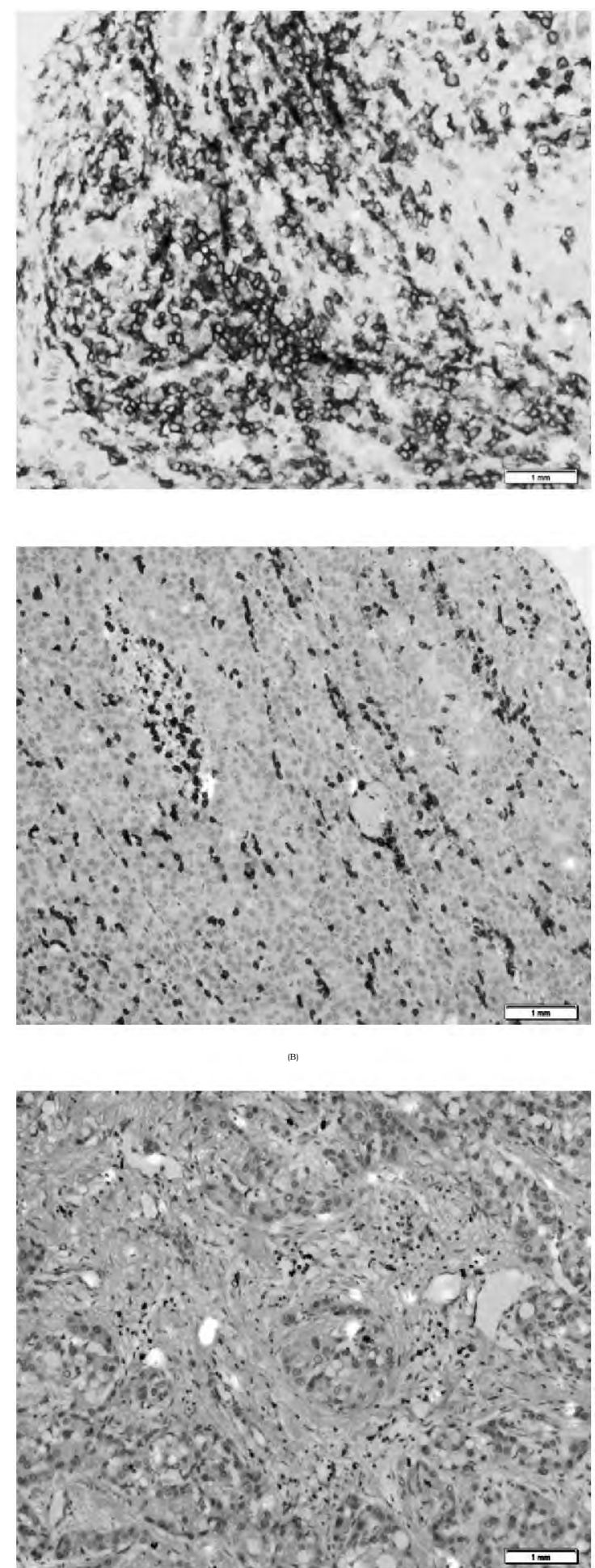

Fig. (1): (A) A case of HCC with positive membranous expression of CD8 T lymphocytes (IHC X400). (B): A case of $\mathrm{HCC}$ with adjacent non cirrhotic liver showing high CD8 percentage of expression (IHC X200). (C): A case of $\mathrm{HCC}$ with adjacent cirrhotic liver showing low CD8 percentage of expression (IHC X200). 
Table (3): Relationship between CD8 percentage of expression and clinicopathological parameters in the studied HCC cases.

\begin{tabular}{|c|c|c|c|c|c|c|}
\hline \multirow{3}{*}{ Variables } & \multicolumn{4}{|c|}{ CD8 percentage } & \multirow{3}{*}{$x^{2}$} & \multirow{3}{*}{$p$} \\
\hline & \multicolumn{2}{|c|}{ Low $(0-20)(n=63)$} & \multicolumn{2}{|c|}{ High $(21-100)(n=49)$} & & \\
\hline & No & $\%$ & No & $\%$ & & \\
\hline $\begin{array}{l}\text { Age: } \\
\quad<60 \\
\quad \geq 60\end{array}$ & $\begin{array}{l}46 \\
17\end{array}$ & $\begin{array}{l}61.3 \\
45.9\end{array}$ & $\begin{array}{l}29 \\
20\end{array}$ & $\begin{array}{l}38.7 \\
54.1\end{array}$ & 2.384 & 0.123 \\
\hline $\begin{array}{l}\text { Gender: } \\
\text { Male } \\
\text { Female }\end{array}$ & $\begin{array}{l}53 \\
10\end{array}$ & $\begin{array}{l}58.2 \\
47.6\end{array}$ & $\begin{array}{l}38 \\
11\end{array}$ & $\begin{array}{l}41.8 \\
63.6\end{array}$ & 0.782 & 0.376 \\
\hline $\begin{array}{l}\text { Etiology: } \\
\text { Viral } \\
\text { Non viarl }\end{array}$ & $\begin{array}{l}59 \\
4\end{array}$ & $\begin{array}{l}58.4 \\
36.4\end{array}$ & $\begin{array}{l}42 \\
7\end{array}$ & $\begin{array}{l}42.3 \\
36.4\end{array}$ & 1.960 & 0.161 \\
\hline $\begin{array}{l}A F P(n g / m l): \\
\quad<200(\mathrm{ng} / \mathrm{ml}) \\
\quad \geq 200(\mathrm{ng} / \mathrm{ml})\end{array}$ & $\begin{array}{l}(\mathrm{n}=52) \\
41 \\
11\end{array}$ & $\begin{array}{l}57.7 \\
55.0\end{array}$ & $\begin{array}{l}(\mathrm{n}=39) \\
30 \\
9\end{array}$ & $\begin{array}{l}57.7 \\
45.0\end{array}$ & 0.048 & 0.826 \\
\hline $\begin{array}{l}\text { Child pugh classification: } \\
\text { A } \\
\text { B } \\
\text { C }\end{array}$ & $\begin{array}{l}(\mathrm{n}=51) \\
36 \\
15 \\
0\end{array}$ & $\begin{array}{l}62.1 \\
50.0 \\
0\end{array}$ & $\begin{array}{l}(\mathrm{n}=37) \\
22 \\
15 \\
0\end{array}$ & $\begin{array}{l}37.9 \\
50.0 \\
0\end{array}$ & 1.182 & 0.277 \\
\hline $\begin{array}{l}\text { Focality: } \\
\text { Single } \\
\text { Multiple }\end{array}$ & $\begin{array}{l}42 \\
21\end{array}$ & $\begin{array}{l}52.5 \\
65.6\end{array}$ & $\begin{array}{l}38 \\
11\end{array}$ & $\begin{array}{l}47.5 \\
34.4\end{array}$ & 1.600 & 0.206 \\
\hline $\begin{array}{l}\text { Site: } \\
\text { Unilobar } \\
\text { Bilobar }\end{array}$ & $\begin{array}{l}59 \\
4\end{array}$ & $\begin{array}{l}55.7 \\
66.7\end{array}$ & $\begin{array}{l}47 \\
2\end{array}$ & $\begin{array}{l}44.3 \\
33.3\end{array}$ & 0.280 & $\begin{array}{l}p= \\
0.697\end{array}$ \\
\hline $\begin{array}{l}\text { Alive/dead: } \\
\text { Alive } \\
\text { Dead }\end{array}$ & $\begin{array}{l}33 \\
30\end{array}$ & $\begin{array}{l}55.0 \\
57.7\end{array}$ & $\begin{array}{l}27 \\
22\end{array}$ & $\begin{array}{l}45.0 \\
42.3\end{array}$ & 0.082 & 0.775 \\
\hline $\begin{array}{l}\text { Treatment: } \\
\text { Interferon } \\
\text { DAA } \\
\text { No }\end{array}$ & $\begin{array}{l}11 \\
33 \\
19\end{array}$ & $\begin{array}{l}45.8 \\
67.3 \\
48.7\end{array}$ & $\begin{array}{l}13 \\
16 \\
20\end{array}$ & $\begin{array}{l}54.2 \\
32.7 \\
51.3\end{array}$ & 4.409 & 0.110 \\
\hline $\begin{array}{l}\text { CLIP prognostic score system: } \\
0 \\
1 \\
2 \\
3 \\
4 \\
5\end{array}$ & $\begin{array}{l}(\mathrm{n}=51) \\
10 \\
24 \\
7 \\
8 \\
2 \\
0\end{array}$ & $\begin{array}{l}50.0 \\
57.1 \\
70.0 \\
72.7 \\
50.0 \\
0.0\end{array}$ & $\begin{array}{l}(\mathrm{n}=37) \\
10 \\
18 \\
3 \\
3 \\
2 \\
1\end{array}$ & $\begin{array}{l}50.0 \\
42.9 \\
30.0 \\
27.3 \\
50.0 \\
100.0\end{array}$ & 3.658 & $\begin{array}{l}p= \\
0.660\end{array}$ \\
\hline $\begin{array}{l}\text { Size: } \\
\quad<5 \mathrm{~cm} \\
\quad \geq 5 \mathrm{~cm}\end{array}$ & $\begin{array}{l}45 \\
18\end{array}$ & $\begin{array}{l}65.2 \\
41.9\end{array}$ & $\begin{array}{l}24 \\
25\end{array}$ & $\begin{array}{l}34.8 \\
58.1\end{array}$ & $5.873 *$ & $0.015^{*}$ \\
\hline $\begin{array}{l}\text { Clear changes: } \\
\text { Yes } \\
\text { No }\end{array}$ & $\begin{array}{l}4 \\
59\end{array}$ & $\begin{array}{l}44.4 \\
57.3\end{array}$ & $\begin{array}{l}5 \\
44\end{array}$ & $\begin{array}{l}55.6 \\
42.7\end{array}$ & 0.554 & $\begin{array}{l}p= \\
0.501\end{array}$ \\
\hline $\begin{array}{l}\text { Grade: } \\
\text { I } \\
\text { II } \\
\text { III } \\
\text { IV }\end{array}$ & $\begin{array}{l}0 \\
44 \\
17 \\
2\end{array}$ & $\begin{array}{l}0.0 \\
57.9 \\
54.8 \\
50.0\end{array}$ & $\begin{array}{l}1 \\
32 \\
14 \\
2\end{array}$ & $\begin{array}{l}100.0 \\
42.1 \\
45.2 \\
50.0\end{array}$ & 1.544 & $\begin{array}{l}p= \\
0.762\end{array}$ \\
\hline $\begin{array}{l}\text { Combined grade: } \\
\text { I/II } \\
\text { III/IV }\end{array}$ & $\begin{array}{l}44 \\
19\end{array}$ & $\begin{array}{l}57.1 \\
54.3\end{array}$ & $\begin{array}{l}33 \\
16\end{array}$ & $\begin{array}{l}42.9 \\
45.7\end{array}$ & 0.080 & 0.778 \\
\hline $\begin{array}{l}\text { Pathological stage: } \\
\text { T1 } \\
\text { T2 } \\
\text { T3 } \\
\text { T4 }\end{array}$ & $\begin{array}{l}21 \\
35 \\
6 \\
1\end{array}$ & $\begin{array}{l}47.7 \\
63.6 \\
50.0 \\
100.0\end{array}$ & $\begin{array}{l}23 \\
20 \\
6 \\
0\end{array}$ & $\begin{array}{l}52.3 \\
36.4 \\
50.0 \\
0.0\end{array}$ & 3.456 & $\begin{array}{l}p= \\
0.292\end{array}$ \\
\hline $\begin{array}{l}\text { Combined pathologic stage: } \\
\text { Early (T1/T2) } \\
\text { Advanced (T3/T4) }\end{array}$ & $\begin{array}{l}56 \\
7\end{array}$ & $\begin{array}{l}56.6 \\
53.8\end{array}$ & $\begin{array}{l}43 \\
6\end{array}$ & $\begin{array}{l}43.4 \\
46.2\end{array}$ & 0.035 & 0.853 \\
\hline $\begin{array}{l}\text { Lymphovascular invasion: } \\
\text { Present } \\
\text { Absent }\end{array}$ & $\begin{array}{l}31 \\
32\end{array}$ & $\begin{array}{l}63.3 \\
50.8\end{array}$ & $\begin{array}{l}18 \\
31\end{array}$ & $\begin{array}{l}36.7 \\
49.2\end{array}$ & 1.742 & 0.187 \\
\hline $\begin{array}{l}\text { Adjacent liver: } \\
\text { Non cirrhotic } \\
\text { Cirrhotic }\end{array}$ & $\begin{array}{l}7 \\
56\end{array}$ & $\begin{array}{l}35.0 \\
60.9\end{array}$ & $\begin{array}{l}13 \\
36\end{array}$ & $\begin{array}{l}65.0 \\
39.1\end{array}$ & $4.468 *$ & $0.035^{*}$ \\
\hline
\end{tabular}

HCC : Hepatocellular Carcinoma.

No : Number.

$\%$ : Percentage.

AFP : Alpha Fetoprotein.

CLIP score: Cancer of the liver Italian program score.

$\chi^{2}:$ Chi square test.

$\chi$
$p_{*}: p$-value for comparing between the studied categories.

DAA: Direct Acting Antiviral. 
Table (4): Univariate overall survival and recurrence of HCC cases according to CD8 expression.

\begin{tabular}{|c|c|c|c|c|}
\hline \multirow{2}{*}{ Variable } & \multicolumn{4}{|c|}{ Overall survival (months) } \\
\hline & Mean survival time & $\mathrm{SE}$ & Log rank & $p$-value \\
\hline \multicolumn{5}{|l|}{ CD8: } \\
\hline Negative & 24.0 & 24.0 & 0.053 & 0.817 \\
\hline Positive & 34.53 & 30.35 & & \\
\hline \multirow{2}{*}{ Variable } & \multicolumn{4}{|c|}{ Recurrence (months) } \\
\hline & Mean recurrence time & SE & Log rank & $p$-value \\
\hline \multicolumn{5}{|l|}{ CD8: } \\
\hline Negative & 18.0 & 0.0 & 0.632 & 0.427 \\
\hline Positive & 9.727 & 0.990 & & \\
\hline
\end{tabular}

\section{Discussion}

Our study showed no correlation between CD8 expression and clinicopathological parameters rather than adjacent non cirrhotic liver tissue and large tumor size. Giuşcă et al., (2015) indicated the difficulty to confirm the direct connection of lymphocytes to the clinical behavior of the tumor [37].

In current study, there was a statistically significant association between high CD8 percentage and adjacent non cirrhotic liver $(p=0.035)$. This agreed with Freeman et al., (2003) who reported that there was no relationship between hepatic lobular infiltrated cytotoxic $\mathrm{T}$ lymphocytes activity and histological evidence of liver damage. These findings indicated that CD8 T lymphocytes may not play a role in occurrence of severe fibrosis of the liver [38] . Furthermore, Desai et al., (2019) demonstrated that HCC that developed in noncirrhotic liver presents at an advanced stage as HCC in non-cirrhotic patients is clinically silent in its early stages because of lack of symptoms and surveillance imaging [39]. Also, approximately $25 \%$ of non-cirrhotic HCC present with extrahepatic metastasis [40]. The recurrence rate of $\mathrm{HCC}$ in non-cirrhotic liver is extremely high [41]. In context of these results, high CD8 expression is associated with bad prognostic parameter such as non-cirrhotic adjacent liver. This may be explained by the immune scape strategies of tumor cells such as immunoediting, downregulation of MHC molecule expression and presence of inhibitory molecules [42].

In our study, there was a significant association between high CD8 percentage and large tumor size $(<5 \mathrm{~cm})(p=0.015)$. This can be explained by depletion of CD8 T lymphocytes during process of inhibition of tumor progression. This opposes AN et al., (2014) who reported that the average numbers of CD8 T cells in the tumor parenchyma and stroma were higher in patients with tumor diameters $<5 \mathrm{~cm}$ than in patients with tumor diameters $>5 \mathrm{~cm}$, so CD8 is involved in HCC diameter control [43] .

On the other hand, our study showed no correlation between $\mathrm{CD} 8$ expression and overall survival or recurrence of the tumor. This agreed with Giuşcă et al., (2015) who showed that the absence of the correlation between TILs and survival in HCC can be due to intense loss of lymphocytes through the proapoptotic processes that develop in the microenvironment of liver parenchyma [37]. Chang et al., (2017) also found that the predictive role of CD8+ TIL for recurrence or survival was not demonstrated [15]. On the other hand, Gabrielson et al., (2016) revealed that a high density of CD8+ cells in the tumor experienced a significant reduction in the rate of HCC recurrence [44] and this difference might be explained by different antibody clones used. However, accumulating evidence has indicated that the activation state of CTLs, instead of just the existence of CTLs, were of great prognostic significance [45-47].

\section{Conclusion:}

This study showed that there is a significant association between high CD8 cytotoxic T lymphocytes expression and bad prognostic parameters such as non-cirrhotic adjacent liver to the tumor and large tumor size, but there is no correlation between CD8 cytotoxic T lymphocytes and overall survival or recurrence.

\section{References}

1- GHARIB A., KARAM R., ABD EL RAHMAN T. and ELSAWY W.: "COX-2 polymorphisms $-765 \mathrm{G} \rightarrow \mathrm{C}$ and $-1195 \mathrm{~A} \rightarrow \mathrm{G}$ and hepatocellular carcinoma risk." Gene, 543: 234-6, 2014.

2- SIEGEL R., MILLER K. and JEMAL A.: "Cancer statistics, 2019." A Cancer Journal for Clinicians, 69 (1): 734, 2019.

3- Who.int.: Cancer. [online] Available at: https://www.who int/news-room/fact-sheets/detail/cancer [Accessed 11 Mar. 2019], 2019.

4- MOKHTAR N., SALAMA A., BADAWY O., KHORSHED E., MOHAMED G., IBRAHIM M. and ABD ELAZIN H. (eds): "Hepatobiliary system tumors, in cancer pathology registry, A 12-year registry 2000-2011." Chapter 10: 161-74, 2016.

5- BAGHDADY I., FOUAD F., SAYED M., SHOAIB A., SALAH Y., ELSHAYEB E. and HASAN A.: "Serum markers for the early detection of hepatocellular carcinoma in patients with chronic viral hepatitis $\mathrm{C}$ infection." M.M.J., 27: 544-50, 2014.

6- ELSAYED H.M., NABIEL Y. and SHETA T.: "IL 12 Gene Polymorphism in Association with Hepatocellular Carci- 
noma in HCV-infected Egyptian Patients.” Immunol Invest., 46: 123-33, 2017.

7- SHIMODA M., MELLODY K. and ORIMO A.: "Carcinoma-associated fibroblasts are a rate-limiting determinant for tumour progression." Seminars in Cell \& Developmental Biology, 21 (1): 19-25, 2010.

8- CHEN F., ZHUANG X., LIN L., YU P., WANG Y., SHI Y., HU G. and SUN Y.: "New horizons in tumor microenvironment biology: Challenges and opportunities." BMC Med., 13: 1, 2015.

9- SINGH S.R., RAMESHWAR P. and SIEGEL P.: "Targeting tumor microenvironment in cancer therapy." Cancer Lett., 380: 203-4, 2016.

10- PATEL P., SCHUTZER S.E. and PYRSOPOULOS N.: "Immunobiology of hepatocarcinogenesis: Ways to go or almost there?" World J. Gastrointest. Pathophysiol., 7 (3): 242-55, 2016.

11-WADA Y., NAKASHIMA O., KUTAMI R., YAMAMOTO O. and KOJIRO M: "Clinicopathological study on hepatocellular carcinoma with lymphocytic infiltration. Hepatology, 27: 407-14, 1998.

12- FUKUZAWA Y., TAKAHASHI K., FURUTA K., TAGAYA T., ISHIKAWA T., WADA K., OMOTO Y., KOJI T. and KAKUMU S.: "Expression of fas/fas ligand (fasL) and its involvement in infiltrating lymphocytes in hepatocellular carcinoma (HCC)." J. Gastroenterol., 36 (10): 681-8, 2001.

13- YAKIREVICH E., LEFEL O., SOVA Y., STEIN A., COHEN O., IZHAK O.B. and RESNICK M.B.: "Activated status of tumour-infiltrating lymphocytes and apoptosis in testicular seminoma." J. Pathol., 196 (1): 67-75, 2002.

14- IKEGUCHI M., SAITO H., KATANO K., TSUJITANI S., MAETA M. and KAIBARA N.: "Correlation between the lymphocytic infiltration of tumors and the proliferative activity of cancer cells from surgically treated esophageal carcinoma." Oncology, 54 (4): 311-7, 1997.

15- CHANG H., JUNG W., KIM A., KIM H., KIM W., KIM J. and KIM B.: "Expression and prognostic significance of programmed death protein 1 and programmed death ligand-1, and cytotoxic T lymphocyte-associated molecule4 in hepatocellular carcinoma." APMIS, 125 (8): 690-8, 2017.

16- YEH M.M., DANIEL H.D. and TORBENSON M.: "Hepatitis C-associated hepatocellular carcinomas in noncirrhotic livers." Mod. Pathol., 23 (2): 276-83, 2010.

17- CHOI J.Y., JUNG S.W., KIM H.Y., KIM M., KIM Y., KIM D.G. and OH E.J.: "Diagnostic value of AFP-L3 and PIVKA-II in hepatocellular carcinoma according to total-AFP." World J. Gastroenterol., 19 (3): 339-46, 2013.

18- PUGH R.N., MURRAY-LYON I.M., DAWSON J.L., PIETRONI M.C. and WILLIAMS R.: "Transection of the oesophagus for bleeding oesophageal varices." $\mathrm{Br}$. J. Surg., 60 (8): 646-9, 1973.

19- CHOLONGITAS E., PAPATHEODORIDIS G.V., VANGELI M., TERRENI N., PATCH D. and BURROUGHS A.K.: "Systematic review: The model for end-stage liver disease--should it replace Child-Pugh's classification for assessing prognosis in cirrhosis?" Aliment. Pharmacol. Ther., 22 (11-12): 1079-89, 2005.
20- KOK B. and ABRALDES J.G.: "Child-Pugh Classification: Time to Abandon?" Semin Liver Dis., 39 (1): 96-103, 2019.

21- HEIMBACH J.K., KULIK L.M., FINN R.S., SIRLIN C.B., ABECASSIS M.M., ROBERTS L.R. and MARRERO J.A.: "AASLD guidelines for the treatment of hepatocellular carcinoma." Hepatology, 67 (1): 358-80, 2018.

22- GUARINO M., SESSA A., COSSIGA V., MORANDO F., CAPORASO N. and MORISCO F.: "Direct-acting antivirals and hepatocellular carcinoma in chronic hepatitis C: A few lights and many shadows." World J. Gastroenterol., 24 (24): 2582-95, 2018.

23- LLOVET J.M. and BRUIX J.: "Prospective validation of the Cancer of the Liver Italian Program (CLIP) score: A new prognostic system for patients with cirrhosis and hepatocellular carcinoma." In Hepatology, 32: 679-80, United States, 2000.

24- TANNUS R.K., ALMEIDA-CARVALHO S.R., LOUREIRO-MATOS C.A., MIZIARA-GONZALEZ A., SALZEDAS-NETTO A.A., SZEJNFELD D. and SOUZA-SILVA I.: "Evaluation of survival of patients with hepatocellular carcinoma: A comparative analysis of prognostic systems." PLoS One, 13 (4): e0194922, 2018.

25- DAI C.Y., LIN C.Y., TSAI P.C., LIN P.Y., YEH M.L., HUANG C.F. and CHEN Y.L.: "Impact of tumor size on the prognosis of hepatocellular carcinoma in patients who underwent liver resection." J. Chin. Med. Assoc., 81 (2): 155-63, 2018

26- EDMONDSON H.A. and STEINER P.E.: "Primary carcinoma of the liver: A study of 100 cases among 48,900 necropsies." Cancer, 7 (3): 462-503, 1954.

27- PIRISI M., LEUTNER M., PINATO D.J., AVELLINI C., CARSANA L., TONIUTTO P. and BOLDORINI R.: "Reliability and reproducibility of the edmondson grading of hepatocellular carcinoma using paired core biopsy and surgical resection specimens." Arch. Pathol. Lab. Med., 134 (12): 1818-22, 2010.

28- MARTINS-FILHO S.N., PAIVA C., AZEVEDO R.S. and ALVES V.A.F.: "Histological Grading of Hepatocellular Carcinoma-A Systematic Review of Literature." Front Med. (Lausanne), 4: 193, 2017.

29- AMIN M.B., EDGE S., GREENE F., BYRD D.R., BROOKLAND R.K., WASHINGTONM.K. and MEYER L.R.: "AJCC Cancer Staging Manual ed.)." Springer, 2017.

30- LI C., YANG W., ZHANG J., ZHENG X., YAO Y., TU K. and LIU Q.: "SREBP-1 has a prognostic role and contributes to invasion and metastasis in human hepatocellular carcinoma." Int. J. Mol. Sci., 15 (5): 7124-38, 2014.

31- HUBER A.R., GONZALEZ R.S., ORLOFF M.S., BARRY C.T. and WHITNEY-MILLER C.L.: "Accuracy of vascular invasion reporting in hepatocellular carcinoma before and after implementation of subspecialty surgical pathology sign-out." Indian J. Pathol. Microbiol., 60 (4): 5014, 2017.

32- GILTNANE J.M. and RIMM D.L.: "Technology insight: Identification of biomarkers with tissue microarray technology." Nat. Clin. Pract. Oncol., 1 (2): 104-11, 2004. 
33- CHANG B., SHEN L., WANG K., JIN J., HUANG T., CHEN Q. and WU P.: "High number of PD-1 positive intratumoural lymphocytes predicts survival benefit of cytokine-induced killer cells for hepatocellular carcinoma patients." Liver Int., 38 (8): 1449-58, 2018.

34- HUANG Y., WANG F.M., WANG T., WANG Y.J., ZHU Z.Y., GAO Y.T. and DU Z.: "Tumor-infiltrating FoxP3+ Tregs and CD8+ T cells affect the prognosis of hepatocellular carcinoma patients." Digestion, 86 (4): 329-37, 2012.

35- HEIMBACH J.K., KULIK L.M., FINN R.S., SIRLIN C.B., ABECASSIS M.M., ROBERTS L.R. and MARRERO J.A.: "AASLD guidelines for the treatment of hepatocellular carcinoma." Hepatology, 67 (1): 358-80, 2018.

36- DAWSON B. \& TRAPP R.G.: "Basic \& Clinical Biostatistics (5 ed.)" New York, United States, McGraw-Hill Education, 2017.

37- GIUSCA $`$ S., WIERZBICKI P., AM ALINEI C., CARUNTU I. and $\mathrm{AV}{ }^{`} \mathrm{AD}$ `ANEI E.: "Comparative analysis of CD4 and CD8 lymphocytes-evidences for different distribution in primary and secondary liver tumors." Folia Histochemica et Cytobiologica, 53 (3): 272-81, 2015.

38- FREEMAN A., PAN Y., HARVEY C., POST J., LAW M., WHITE P., RAWLINSON W., LLOYD A., MARINOS G. and FFRENCH R.: "The presence of an intrahepatic cytotoxic $\mathrm{T}$ lymphocyte response is associated with low viral load in patients with chronic hepatitis $\mathrm{C}$ virus infection.” Journal of Hepatology, 38 (3): 349-56, 2003.

39- DESAI A., SANDHU S., LAI J. and SANDHU D.: "Hepatocellular carcinoma in non-cirrhotic liver: A comprehensive review." World Journal of Hepatology, 11 (1): $1-18,2019$.

40- SCHÜTTE K., SCHULZ C., PORANZKE J., ANTWEILER K., BORNSCHEIN J., BRETSCHNEIDER T. , AREND J., RICKE J. and MALFERTHEINER P. : "Characterization and prognosis of patients with hepatocellular carcinoma (HCC) in the non-cirrhotic liver." BMC Gastroenterol., 14: 117, 2014.
41- ADAM R., BISMUTH H., CASTAING D., WAECHTER F., NAVARRO F., ABASCAL A., MAJNO P. and ENGERRAN L.: "Repeat hepatectomy for colorectal liver metastases." Ann. Surg., 225: 51-60, 1997.

42- BÜTTNER N., SCHMIDT N. and THIMME R.: "Perspectives of immunotherapy in hepatocellular carcinoma (HCC)." Zeitschrift für Gastroenterologie, 54 (12): 133442, 2016.

43- AN J., JI Q., AN J., MASUDA S. and TSUNEYAMA K.: "Clinicopathological analysis of CD8-positive lymphocytes in the tumor parenchyma and stroma of hepatocellular carcinoma." Oncology Letters, 8 (5): 2284-90, 2014.

44- GABRIELSON A., WU Y., WANG H., JIANG J., KALLAKURY B., GATALICA Z., REDDY S., KLEINER D., FISHBEIN T., JOHNSON L., ISLAND E., SATOSKAR R., BANOVAC F., JHA R., KACHHELA J., FENG P., ZHANG T., TESFAYE A., PRINS P., LOFFREDO C., MARSHALL J., WEINER L., ATKINS M. and HE A.: "Intratumoral CD3 and CD8 T-cell Densities Associated with Relapse-Free Survival in HCC." Cancer Immunology Research, 4 (5): 419-30, 2016.

45- SCHUMACHER K., HAENSCH W., RÖEFZAAD C. and SCHLAG P.M.: "Prognostic significance of activated CD8(+) T cell infiltrations within esophageal carcinomas." Cancer Research, 61 (10): 3932-6, 2001.

46- GRABENBAUER G.G., LAHMER G., DISTEL L. and NIEDOBITEK G.: "Tumor-infiltrating cytotoxic T cells but not regulatory $\mathrm{T}$ cells predict outcome in anal squamous cell carcinoma." Clin. Cancer Res., 12 (11 Pt 1): 3355-60, 2006.

47- VAN BEEK J., ZUR HAUSEN A., SNEL S.N., BERKHOF J., KRANENBARG E.K., VAN De VELDE C.J. and BLOEMENA E.: "Morphological evidence of an activated cytotoxic T-cell infiltrate in EBV-positive gastric carcinoma preventing lymph node metastases." Am. J. Surg. Pathol., 30 (1): 59-65, 2006. 


\section{دور الخلايا التائية (سى دى م) فى سرطان الحخلايا الكبدية،

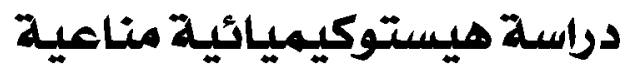

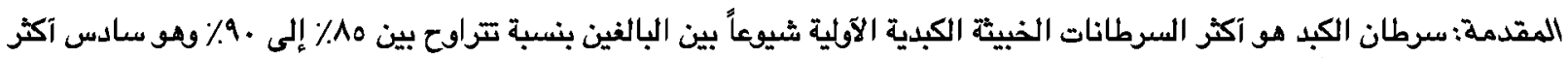

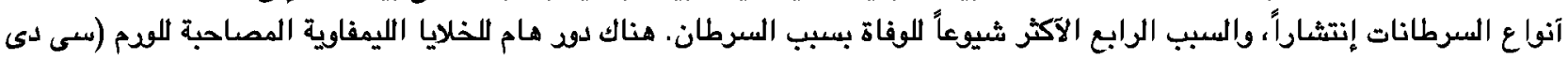

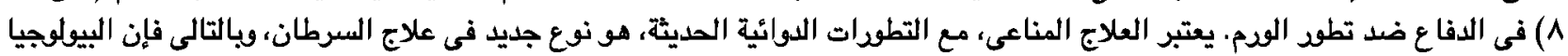
المناعية لتسرطن الخاعلايا الكبدية قيد التحقيق.

الآهداف:دراسة تعبير الخلايا التائية (سى دى ^) فى سرطان الخلايا الكبدية وعلاقتها بالمؤثُرات السريرية والمرضية وإكتشاف تآثيرها على تطود سرطان الخلايا الكبدية.

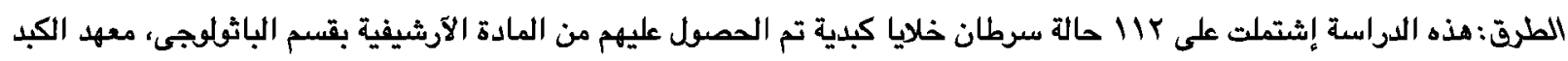

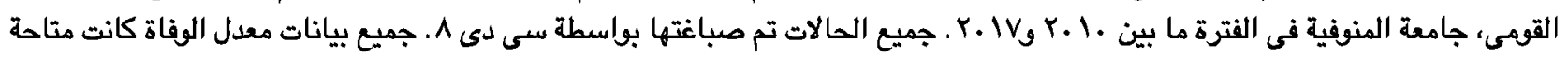
لجميع حالات سرطان الخلايا الكبدية.

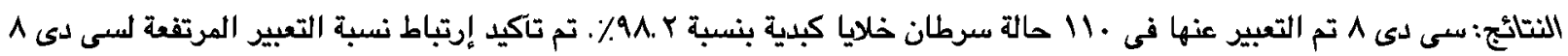

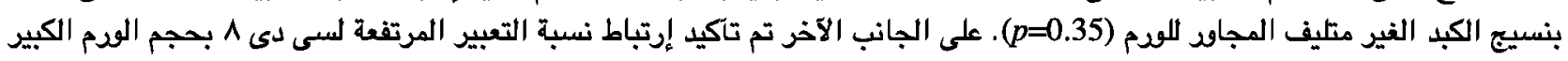

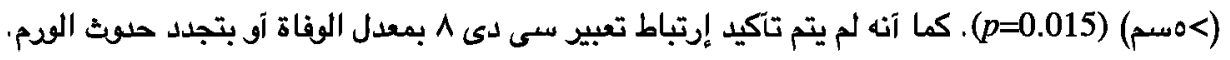

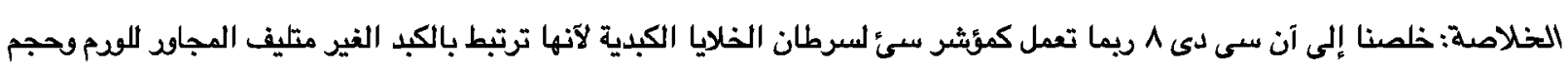

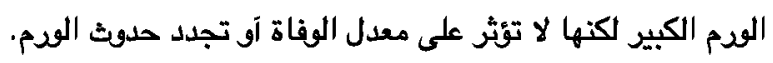

